\title{
Analysis and Design of Adaptive Synchronization of a Complex Dynamical Network with Time-Delayed Nodes and Coupling Delays
}

\author{
Yu Miao, ${ }^{1,2}$ He Liang, ${ }^{1,2}$ Zhao Haiyun, ${ }^{1,2}$ Chen Zhigang, ${ }^{1,2}$ and Yi Junyan ${ }^{3}$ \\ ${ }^{1}$ School of Mechanical-Electronic and Automobile Engineering, Beijing University of Civil Engineering and Architecture, \\ Beijing 100044, China \\ ${ }^{2}$ Beijing Key Laboratory of Service Performance of Urban Rail Transit Vehicles, Beijing University of Civil Engineering and Architecture, \\ Beijing 100044, China \\ ${ }^{3}$ School of Electrical and Information Engineering, Beijing University of Civil Engineering and Architecture, Beijing 100044, China
}

Correspondence should be addressed to Yu Miao; olivermiaoer@163.com

Received 7 April 2017; Revised 28 August 2017; Accepted 19 September 2017; Published 19 October 2017

Academic Editor: Alessandro Lo Schiavo

Copyright (C) 2017 Yu Miao et al. This is an open access article distributed under the Creative Commons Attribution License, which permits unrestricted use, distribution, and reproduction in any medium, provided the original work is properly cited.

\begin{abstract}
This paper is devoted to the study of synchronization problems in uncertain dynamical networks with time-delayed nodes and coupling delays. First, a complex dynamical network model with time-delayed nodes and coupling delays is given. Second, for a complex dynamical network with known or unknown but bounded nonlinear couplings, an adaptive controller is designed, which can ensure that the state of a dynamical network asymptotically synchronizes at the individual node state locally or globally in an arbitrary specified network. Then, the Lyapunov-Krasovskii stability theory is employed to estimate the network coupling parameters. The main results provide sufficient conditions for synchronization under local or global circumstances, respectively. Finally, two typical examples are given, using the M-G system as the nodes of the ring dynamical network and second-order nodes in the dynamical network with time-varying communication delays and switching communication topologies, which illustrate the effectiveness of the proposed controller design methods.
\end{abstract}

\section{Introduction}

A complex network refers to a large-scale network with a complex topology and dynamic behavior. It consists of a large set of interconnected nodes, in which the node is a fundamental unit with specific contents [1]. Complex networks exist in many fields of science, engineering, and society and have attracted much attention in recent years [2-5]. As one of the most important collective behaviors, synchronization phenomena have been a topic of research. There are a lot of synchronization phenomena in physical sciences and in mathematics. Several books and reviews [6-9] have also dealt with this topic. Such applications are pervasive and include clock synchronization in complex networks [1012], coordination of unmanned aerial vehicles [13], and fair allocation of network resources [14].
In the past decade, special attention has been paid to the synchronization of dynamical systems, particularly largescale and complex networks of chaotic oscillators $[15,16]$. With the discovery of the small-world effect and scale-free feature of most complex networks, a lot of works have been concentrated on synchronization in small-world networks and scale-free networks [17-20]. In these investigations, an essential requirement is that the structure of the networks and the coupling functions are known beforehand. In order to overcome the aforementioned constraints, synchronization in complex networks by controller methods has also been investigated [21-24]. Zhou et al. [21] proposed some synchronization criteria and designed simple controllers for several specific complex networks. In [22], for a class of complex networks with uncertain inner couplings, the event-triggered synchronization control problem was put 
forward. The uncertain inner coupling under consideration was characterized in terms of the interval matrix. In order to save the communication and computation resources, the event-based mechanism was adopted and an eventtriggered synchronization control scheme was proposed for the complex networks. Based on the leader-follower model, Zhou et al. [23] proposed an improved network structure model for realizing the cluster synchronization on multiple subnetworks of complex networks, and some suitable pinning controllers on the chosen nodes of each follower's subnetwork are designed. Yu et al. [24] employed a decomposition approach to incorporate the nodes' inertial effects into the distributed control design for second-order nodes in a dynamical network with communication delays and switching communication topologies.

However, the following problems still exist in the control of dynamical networks and have not been solved in a good way. It is often difficult to get an exact estimation of coupling coefficients in reality. Therefore, it is preferable if the coupling functions in a dynamical network are not restricted to be completely known. If the network structure is only partially known or completely unknown, it is very difficult or even impossible to design a controller to achieve the intended network synchronization. Besides the problem above, the nodes in the dynamical network usually have the characteristics of time delay. Because of the finite speeds of transmission as well as traffic congestion, a signal or influence traveling through a complex network is often associated with time delays. Time delay is the main factor which gives rise to instability in dynamical networks. This issue has attracted the attention of many scholars recently. In $[25,26]$, the authors found that the solution space of time-delayed networks was infinitedimensional, and its theoretical analysis was often very difficult. Dhamala et al. [27] assumed that the coupling was linear between nodes and explored the effect on the synchronization of time-delayed nodes in various networks. Then, Atay et al. [28] found that altering the time delay would be helpful to improve the capability of the network synchronization. Gao et al. [29] studied the uncertain time-delay systems and robust stability analysis. They solved the problems about a class of nonlinear time-delay systems with memory stabilization. In [30], a complex dynamical network model with time-varying inner coupling was introduced. And a sufficient condition was given to achieve the exponential synchronization.

In this paper, we will give positive answers to all the questions above. We combine the adaptive control method with the network with time-delayed nodes and coupling delays. The local and global features of adaptive synchronization of networks are studied based on the LyapunovKrasovskii stability theorem. The results we got are suitable for the general dynamical network; that is, they are independent of the network topology. The paper is organized as follows. The general network model and several mathematical preliminaries are described in Section 2. In Section 3, the analysis of adaptive synchronization is studied, and also two desirable controllers are designed. Section 4 gives two typical examples. The M-G system is used with the timedelayed nodes in the ring network and second-order nodes in the dynamical network with time-varying communication delays and switching communication topologies. Simulation results show the effectiveness and correctness of the proposed methods. Finally, conclusions are given in Section 5.

\section{Network Model and Mathematical Preliminaries}

2.1. A Complex Dynamical Network Model with Time-Delayed Nodes and Coupling Delays. Consider a nonlinear complex network consisting of $N$ identical delay nodes, which are coupled as follows [31]:

$$
\begin{aligned}
\dot{x}_{i}=f\left(x_{i}, t\right)+g_{i}\left(x_{1}, x_{2}, \ldots, x_{N}\right)+u_{i}(t), & \\
& t \geq 0,1 \leq i \leq N,
\end{aligned}
$$

where $x_{i}=\left(x_{i 1}, x_{i 2}, \ldots, x_{i n}\right)^{\mathrm{T}} \in \mathbb{R}^{n}$ represents the state vector of the $i$ th node; $f: \Omega \times \mathbb{R}^{+} \rightarrow \mathbb{R}^{n}$ represents a smooth nonlinear vector function; $g_{i}: \mathbb{R}^{m} \rightarrow \mathbb{R}^{n}, m=n N$ represents a smooth unknown nonlinear coupling function; $u_{i}(t) \in \mathbb{R}^{n}, 1 \leq i \leq N$, represents the control input.

When the network reaches synchronization, that is, $t \rightarrow$ $\infty, x_{1}(t)=x_{2}(t)=\cdots=x_{N}(t)$, the coupling control will disappear at this point; that is,

$$
g_{i}\left(x_{1}, x_{2}, \ldots, x_{N}\right)+u_{i}(t)=0 .
$$

This ensures that the solution of any solitary node $x_{i}(t)$ is also the solution of the synchronous coupling network.

As can be seen from the Introduction, there is a delay in the actual network. After adding the time-delay term in the model of (1), the dynamical network with these time-delayed nodes and coupling delays can be written as

$$
\begin{aligned}
\dot{x}_{i}= & f_{1}\left(x_{i}, t\right)+f_{2}\left(x_{i}(t-\tau), t\right) \\
& +g_{i}\left(x_{1}(t-\tau), x_{2}(t-\tau), \ldots, x_{N}(t-\tau)\right) \\
& +u_{i}(t),
\end{aligned}
$$

where $x_{i}=\left(x_{i 1}, x_{i 2}, \ldots, x_{i n}\right)^{\mathrm{T}} \in \mathbb{R}^{n}$ represents the state vector of the $i$ th node; $f_{k}: \Omega \times \mathbb{R}^{+} \rightarrow \mathbb{R}^{n}, k=1,2$, represents a continuous nonlinear function; $g_{i}: \mathbb{R}^{m} \rightarrow \mathbb{R}^{n}, m=$ $n N$, represents an unknown continuous nonlinear coupling function; $\tau \geq 0$ is a time delay; and $u_{i}(t) \in \mathbb{R}^{n}, 1 \leq i \leq N$, represents a control input.

Obviously, when the complex network achieves synchronization, there is $x_{1}(t)=x_{2}(t)=\cdots=x_{N}(t)$, and the coupling and control items will disappear at this time; that is, $g_{i}\left(x_{1}(t-\tau), x_{2}(t-\tau), \ldots, x_{N}(t-\tau)\right)+u_{i}(t)=0$.

2.2. Mathematical Preliminaries. The following definitions and assumptions are necessary for discussing the network synchronization problem.

Definition 1. Let $\xi(t)$ be a unique solution of a complex network (3) which satisfies the following equation:

$$
\dot{\xi}(t)=f_{1}(\xi(t), t)+f_{2}(\xi(t-\tau), t),
$$

where $\xi(t)$ can be an equilibrium point, a nontrivial periodic orbit, or even a chaotic orbit. 
Definition 2. If there is a nonempty subset $D \subseteq \mathbb{R}$, for any $t \geq 0, \tau \geq 0$, there is $\xi(t) \in \mathbb{R}$, which satisfies the following formula:

$$
\lim _{t \rightarrow \infty}\left\|x_{i}(t)-\xi(t)\right\|_{2}=0, \quad 1 \leq i \leq N .
$$

Then, the solution of the complex network (3) is asymptotically synchronized with (5). $D \times \cdots \times D$ is called the synchronous region.

Next, subtracting (2) from (4) yields the following error dynamical system:

$$
\begin{aligned}
\dot{e}_{i}(t)= & \bar{f}_{1}\left(x_{i}, \xi\right)+\bar{f}_{2}\left(x_{i}(t-\tau), \xi\right) \\
& +\bar{g}_{i}\left(x_{1}(t-\tau), x_{2}(t-\tau), \ldots, x_{N}(t-\tau), \xi\right) \\
& +u_{i}(t)
\end{aligned}
$$

where

$$
\begin{aligned}
& \bar{g}_{i}\left(x_{1}(t-\tau), x_{2}(t-\tau), \ldots, x_{N}(t-\tau), \xi\right) \\
& =g_{i}\left(x_{1}(t-\tau), x_{2}(t-\tau), \ldots, x_{N}(t-\tau), \xi\right) \\
& \quad-g_{i}(\xi, \xi, \ldots, \xi, \xi), \\
& \bar{f}_{1}\left(x_{i}, \xi\right)=f_{1}\left(x_{i}, t\right)-f_{1}(\xi, t), \\
& \bar{f}_{2}\left(x_{i}(t-\tau), \xi\right)=f_{2}\left(x_{i}(t-\tau), t\right)-f_{2}(\xi(t-\tau), t) .
\end{aligned}
$$

By linearizing (6) which is evaluated at $\xi(t, \tau)$, we can get the following equation:

$$
\begin{aligned}
\dot{e}_{i}(t)= & A(t) e_{i}(t)+B(t) e_{i}(t-\tau) \\
& +\bar{g}_{i}\left(x_{1}(t-\tau), x_{2}(t-\tau), \ldots, x_{N}(t-\tau), \xi\right) \\
& +u_{i}(t)
\end{aligned}
$$

where $\|A(t)\|_{2}=\left\|D f_{1}(\xi, t)\right\|_{2},\|B(t)\|_{2}=\left\|D f_{2}(\xi, t)\right\|_{2}, A(t)$ is a Jacobian matrix of $f_{1}$ on $\xi(t), B(t)$ is a Jacobian matrix of $f_{2}$ on $\xi(t)$, and there is $e_{i}(t)=x_{i}(t)-\xi(t)$.

In order to facilitate the follow-up study, we present the following assumptions.

Assumption 3. Suppose that $\|A(t)\|_{2}$ and $\|B(t)\|_{2}$ are bounded. That is, there exist nonnegative constants $\alpha$ and $\beta$ satisfying $\|A(t)\|_{2} \leq \alpha$ and $\|B(t)\|_{2} \leq \beta$.

Assumption 4. Suppose that the nonlinear function $g(\cdot)$ is Lipschitz continuous. That is, $\forall x_{i}, x_{j} \in \mathbb{R}^{n}$, the following formula holds:

$$
\left\|g\left(x_{i}, t\right)-g\left(x_{j}, t\right)\right\|_{2} \leq \mu_{i j}\left\|x_{i}(t)-x_{j}(t)\right\|_{2} .
$$

Therefore, (3) can be written as follows:

$$
\begin{aligned}
& \left\|\bar{g}_{i}\left(x_{1}(t-\tau), x_{2}(t-\tau), \ldots, x_{N}(t-\tau), \xi\right)\right\|_{2} \\
& \quad \leq \sum_{j=1}^{N} \mu_{i j}\left\|e_{j}(t-\tau)\right\|_{2},
\end{aligned}
$$

where $\mu_{i j}$ is a nonnegative Lipschitz constant, $1 \leq i \leq N, 1 \leq$ $j \leq N$.
Lemma 5 (see [32]). For any $\omega, \zeta \in R^{n}, \rho>0$, one has $2 \omega^{T} \zeta \leq$ $\rho \omega^{T} \omega+(1 / \rho) \zeta^{T} \zeta$.

\section{Synchronization Analysis and Design of Adaptive Controller}

In this section, we apply the Lyapunov-Krasovskii stability theorem to the problems of local and global synchronization of coupled delay dynamical network with time-delay nodes. Then, we design an adaptive controller according to the different situations.

3.1. Local Synchronization of Coupled Delay Dynamical Network with Time-Delay Nodes. Consider all the nodes in the dynamical network without loss of energy and generality. Assume that the $i$ th $(1 \leq i \leq N)$ node is controlled by the following adaptive controller, which is described by

$$
\begin{aligned}
u_{i}(t) & =-\varsigma_{i} e_{i}(t), \\
\dot{\zeta}_{i} & =k_{i}\left\|e_{i}(t)\right\|_{2}^{2},
\end{aligned}
$$

where $\varsigma_{i}$ and $k_{i}, i=1,2, \ldots, N$, are positive constants. Thus, the controlled network (2) can be rewritten as follows:

$$
\begin{aligned}
\dot{x}_{i}= & f_{1}\left(x_{i}, t\right)+f_{2}\left(x_{i}(t-\tau), t\right) \\
& +g_{i}\left(x_{1}(t-\tau), x_{2}(t-\tau), \ldots, x_{N}(t-\tau)\right) \\
& -\varsigma_{i} e_{i}(t), \\
\dot{\zeta}_{i}= & k_{i}\left\|e_{i}(t)\right\|_{2}^{2}, \\
\dot{e}_{i}(t)= & A(t) e_{i}+B(t) e_{i}(t-\tau) \\
& +\bar{g}_{i}\left(x_{1}(t-\tau), x_{2}(t-\tau), \ldots, x_{N}(t-\tau)\right) \\
& -\varsigma_{i} e_{i}(t), \\
\dot{\zeta}_{i}= & k_{i}\left\|e_{i}(t)\right\|_{2}^{2} .
\end{aligned}
$$

When the parameters $\mu_{i j}, \quad(1 \leq i \leq N, 1 \leq j \leq N)$ are known or unknown but bounded nonlinear couplings, there exists the following theorem.

Theorem 6. If Assumptions 3 and 4 are established, then the complex network (2) will achieve local asymptotical stability under the action of the adaptive controller (11).

Proof. Construct a Lyapunov candidate as follows:

$$
\begin{aligned}
V(e, t, \tau)= & \frac{1}{2} \sum_{i=1}^{N} e_{i}^{\mathrm{T}}(t) e_{i}(t)+\sum_{i=1}^{N} \int_{t-\tau}^{t} e_{i}^{\mathrm{T}}(s) \mathrm{H} e_{i}(s) d s \\
& +\frac{1}{2} \sum_{i=1}^{N} \frac{\left(\varsigma_{i}-\widehat{\varsigma}_{i}\right)^{2}}{k_{i}}
\end{aligned}
$$

where $e(t)=\left(\left\|e_{1}(t)\right\|,\left\|e_{2}(t)\right\|, \ldots,\left\|e_{N}(t)\right\|,\left\|e_{1}(t-\tau)\right\|, \| e_{2}(t-\right.$ $\left.\tau)\|, \ldots,\| e_{N}(t-\tau) \|\right)^{\mathrm{T}}, \widehat{\varsigma}_{i}$ and $k_{i}, i=1,2, \ldots, N$, are positive constants, and $\mathrm{H} \in \mathbb{R}^{n \times n}$ is the positive definite matrix that 
needs to be determined. Thus, the time derivative of $V(e, t, \tau)$ along (6) is

$$
\begin{aligned}
\dot{V} & (e, t, \tau)=\frac{1}{2} \sum_{i=1}^{N}\left(\dot{e}_{i}^{\mathrm{T}}(t) e_{i}(t)+e_{i}^{\mathrm{T}}(t) \dot{e}_{i}(t)\right) \\
& +\sum_{i=1}^{N}\left[e_{i}^{\mathrm{T}}(t) \mathrm{H} e_{i}(t)-e_{i}^{\mathrm{T}}(t-\tau) \mathrm{H} e_{i}(t-\tau)\right] \\
& -\sum_{i=1}^{N} \frac{\left(\varsigma_{i}-\widehat{\varsigma}_{i}\right) \dot{\zeta}_{i}}{k_{i}}=\frac{1}{2} \sum_{i=1}^{N}\left[\left(A(t) e_{i}(t)\right.\right. \\
& +B(t) e_{i}(t-\tau) \\
& +g_{i}\left(x_{1}(t-\tau), x_{2}(t-\tau), \ldots, x_{N}(t-\tau), \xi\right) \\
& \left.+u_{i}(t)\right)^{\mathrm{T}} e_{i}(t)+e_{i}^{\mathrm{T}}(t)\left(A(t) e_{i}(t)+B(t) e_{i}(t-\tau)\right. \\
& +g_{i}\left(x_{1}(t-\tau), x_{2}(t-\tau), \ldots, x_{N}(t-\tau), \xi\right) \\
& \left.\left.+u_{i}(t)\right)\right]+\sum_{i=1}^{N}\left[e_{i}^{\mathrm{T}}(t) \mathrm{H} e_{i}(t)-e_{i}^{\mathrm{T}}(t-\tau) \mathrm{H} e_{i}(t\right. \\
& -\tau)]-\sum_{i=1}^{N} \frac{\left(\varsigma_{i}-\widehat{\varsigma}_{i}\right) \dot{\zeta}_{i}}{k_{i}}=\frac{1}{2} \sum_{i=1}^{N} e_{i}^{\mathrm{T}}(t)\left[A^{\mathrm{T}}(t)+A(t)\right. \\
& +\tau)-\sum_{i=1}^{N} \frac{\left(\varsigma_{i}-\widehat{\zeta}_{i}\right) \dot{\zeta}_{i}}{k_{i}} . \\
& +2 \mathrm{H}] e_{i}(t)+\sum_{i=1}^{N}\left[e_{i}^{\mathrm{T}}(t-\tau) B^{\mathrm{T}}(t) e_{i}(t)+e_{i}^{\mathrm{T}}(t) B(t)\right. \\
& \left.+e_{i}(t-\tau)\right]+\sum_{i=1}^{N} e_{i}^{\mathrm{T}}(t) g_{i}\left(x_{1}(t-\tau), x_{2}(t-\tau), \ldots,\right. \\
&
\end{aligned}
$$

Substituting the adaptive controller (11) into (14) yields the following:

$$
\begin{aligned}
& \dot{V}(e, t, \tau)=\sum_{i=1}^{N} e_{i}^{\mathrm{T}}(t)\left[\frac{A^{\mathrm{T}}(t)+A(t)}{2}+\mathrm{H}\right] e_{i}(t) \\
& +\sum_{i=1}^{N}\left[e_{i}^{\mathrm{T}}(t-\tau) B^{\mathrm{T}}(t) e_{i}(t)+e_{i}^{\mathrm{T}}(t) B(t) e_{i}(t-\tau)\right] \\
& +\sum_{i=1}^{N} e_{i}^{\mathrm{T}}(t) \\
& +g_{i}\left(x_{1}(t-\tau), x_{2}(t-\tau), \ldots, x_{N}(t-\tau), \xi\right) \\
& +\sum_{i=1}^{N} \varsigma_{i} e_{i}^{\mathrm{T}}(t) e_{i}(t)-\sum_{i=1}^{N} e_{i}^{\mathrm{T}}(t-\tau) \mathrm{H} e_{i}(t-\tau)
\end{aligned}
$$

$$
\begin{aligned}
& -\sum_{i=1}^{N}\left(\varsigma_{i}-\widehat{\varsigma}_{i}\right) e_{i}^{\mathrm{T}}(t) e_{i}(t)=\sum_{i=1}^{N} e_{i}^{\mathrm{T}}(t)\left[\frac{A^{\mathrm{T}}(t)+A(t)}{2}\right. \\
& \left.+\mathrm{H}-\widehat{\varsigma}_{i} I_{n \times n}\right] e_{i}(t) \\
& +\sum_{i=1}^{N}\left[e_{i}^{\mathrm{T}}(t-\tau) B^{\mathrm{T}}(t) e_{i}(t)+e_{i}^{\mathrm{T}}(t) B(t) e_{i}(t-\tau)\right] \\
& -\sum_{i=1}^{N} e_{i}^{\mathrm{T}}(t-\tau) \mathrm{H} e_{i}(t-\tau)+\sum_{i=1}^{N} e_{i}^{\mathrm{T}}(t) \\
& \cdot g_{i}\left(x_{1}(t-\tau), x_{2}(t-\tau), \ldots, x_{N}(t-\tau), \xi\right) .
\end{aligned}
$$

According to Assumptions 3 and 4 and Lemma 5, selecting $\mathrm{H}=\varepsilon I_{n \times n}$, where the constant $\varepsilon>0$, it can be obtained that

$$
\begin{aligned}
& \dot{V}(e, t, \tau) \leq \sum_{i=1}^{N} e_{i}^{\mathrm{T}}(t)\left[\frac{A^{\mathrm{T}}(t)+A(t)}{2}+\left(\varepsilon-\widehat{\varsigma}_{i}\right) I_{n \times n}\right] \\
& \cdot e_{i}(t)+\frac{1}{2} \sum_{i=1}^{N}\left[e_{i}^{\mathrm{T}}(t-\tau)\left(B^{\mathrm{T}} B+I\right) e_{i}(t-\tau)\right. \\
& \left.+e_{i}^{\mathrm{T}}(t)\left(B^{\mathrm{T}} B+I\right) e_{i}(t)\right] \\
& +\sum_{i=1}^{N} \sum_{j=1}^{N} \mu_{i j}\left\|e_{i}(t)\right\|_{2}\left\|e_{j}(t-\tau)\right\|_{2}-\sum_{i=1}^{N} e_{i}^{\mathrm{T}}(t-\tau) \\
& \cdot+e_{i}(t-\tau) \leq \sum_{i=1}^{N}\left(\alpha+\varepsilon+\beta^{2}+1-\widehat{\varsigma}_{i}\right)\left\|e_{i}(t)\right\|_{2}^{2} \\
& +\sum_{i=1}^{N} \sum_{j=1}^{N} \mu_{i j}\left\|e_{i}(t)\right\|_{2}\left\|e_{j}(t-\tau)\right\|_{2}-\frac{\beta^{2}-\varepsilon+1}{2} \\
& \quad \cdot \sum_{i=1}^{N}\left\|e_{i}(t-\tau)\right\|_{2}^{2}=e^{\mathrm{T}}(t)\left(\operatorname { d i a g } \left\{\alpha+\varepsilon+\beta^{2}+1\right.\right. \\
& -\widehat{\varsigma}_{1}, \ldots, \alpha+\varepsilon+\beta^{2}+1-\widehat{\varsigma}_{N},-\frac{\beta^{2}-\varepsilon+1}{2}, \ldots \\
& \left.\left.\quad-\frac{\beta^{2}-\varepsilon+1}{2}\right\}+P\right) e(t),
\end{aligned}
$$

where $=\left(\begin{array}{cc}0 & \Gamma / 2 \\ \Gamma / 2 & 0\end{array}\right), \Gamma=\left(\mu_{i j}\right)_{N \times N}$.

Therefore, we can choose the appropriate constants $\widehat{\varsigma}_{i}, i=$ $1,2, \ldots, N$, and $\varepsilon>0$, such that the diagonal matrix $\operatorname{diag}\{\alpha+$ $\varepsilon+\beta^{2}+1-\widehat{\varsigma}_{1}, \ldots, \alpha+\varepsilon+\beta^{2}+1-\widehat{\varsigma}_{N},-\left(\beta^{2}-\varepsilon+1\right) / 2, \ldots,-\left(\beta^{2}-\varepsilon+\right.$ 1) $/ 2\}+P$ is a negative matrix. This equation shows that $c_{i}, i=$ $1,2, \ldots, N$, is uniformly bounded, and the error system (8) is asymptotically stable by the action of the adaptive controller (11). By using the Lyapunov method, it can be clearly seen that the error system (6) is also asymptotically stable under the action of the adaptive controller (11). It is concluded that $e=0$ is an asymptotically stable equilibrium point of the error system (8) and it is also deduced that $e=0$ is an asymptotically stable equilibrium point of the system. 
The above stability criteria are delay-independent. In a coupled complex network with different delays, (17) can also be synchronized using an adaptive controller (11):

$$
\begin{aligned}
\dot{x}_{i}= & f_{1}\left(x_{i}, t\right)+f_{2}\left(x_{i}(t-\tau), t\right) \\
& +g_{i}\left(x_{1}\left(t-\tau_{1}\right), x_{2}\left(t-\tau_{2}\right), \ldots, x_{N}\left(t-\tau_{N}\right)\right) \\
& +u_{i}(t),
\end{aligned}
$$

where $\tau_{i}>0, i=1,2, \ldots, N$, is the coupling delay; the proof is similar to that of Theorem 6 . We can derive from Theorem 6 that synchronization of such complex networks mainly depends on three basic parameters: the dynamic characteristics of complex network nodes $(\alpha, \beta, \varepsilon)$, the dynamic parameters of the adaptive controller $(\zeta)$, and the network structure parameters $\left(\mu_{i j}\right)$.

In summary, we can see that the dynamical network with time-delay nodes and coupling delay structure can achieve local synchronization; that is, complex network synchronization can be realized under the action of adaptive controller (11).

\subsection{Global Synchronization of Coupled Delay Dynamical} Network with Time-Delay Nodes. In this section, we discuss the global synchronization problem with time-delay nodes and coupled delay networks. The network structure is as follows:

$$
\begin{aligned}
\dot{x}_{i}= & f_{1}\left(x_{i}, t\right)+f_{2}\left(x_{i}(t-\tau), t\right)+h_{1}\left(x_{i}, t\right) \\
& +h_{2}\left(x_{i}(t-\tau), t\right) \\
& +g_{i}\left(x_{1}(t-\tau), x_{2}(t-\tau), \ldots, x_{N}(t-\tau)\right) \\
& +u_{i}(t),
\end{aligned}
$$

where $h_{i}: \mathbb{R}^{n} \times \mathbb{R}^{+} \rightarrow \mathbb{R}^{n}, i=1,2$, is an unknown and smooth function. According to the derivation process of Section 2, we can compute the error dynamic equation of (11):

$$
\begin{aligned}
\dot{e}_{i}(t)= & A(t) e_{i}(t)+B(t) e_{i}(t-\tau)+h_{1}\left(x_{i}, \xi\right) \\
& +h_{2}\left(x_{i}(t-\tau), \xi\right) \\
& +g_{i}\left(x_{1}(t-\tau), x_{2}(t-\tau), \ldots, x_{N}(t-\tau)\right) \\
& +u_{i}(t)
\end{aligned}
$$

where $h_{1}\left(x_{i}, \xi\right)=h_{1}\left(x_{i}, t\right)-h_{1}(\xi, t), h_{2}\left(x_{i}(t-\tau), \xi\right)=h_{2}\left(x_{i}(t-\right.$ $\tau), t)-h_{2}(\xi, t)$.

The definitions of other sections are the same as those defined in Section 2.

Assumption 7 (see [33]). Suppose that there are unknown but nonnegative constants $\gamma_{i}, i=1,2, \ldots, N$, satisfying

$$
\begin{gathered}
\left\|h_{1}\left(x_{i}, \xi\right)\right\|_{2} \leq \gamma_{i}\left\|e_{i}(t)\right\|_{2}, \\
\left\|h_{2}\left(x_{i}(t-\tau), \xi\right)\right\|_{2} \leq \gamma_{i}\left\|e_{i}(t-\tau)\right\|_{2}, \\
i=1,2, \ldots, N . N
\end{gathered}
$$

The following theorem gives a method of global synchronization criterion for the dynamical network (18).
Theorem 8. If Assumptions 3, 4, and 7 are established, then the complex dynamical network (18) achieves global asymptotic stability under the action of the adaptive controller of (11).

Proof. Select the following Lyapunov function:

$$
\begin{aligned}
V(e, t, \tau)= & \frac{1}{2} \sum_{i=1}^{N} e_{i}^{\mathrm{T}}(t) e_{i}(t)+\sum_{i=1}^{N} \int_{t-\tau}^{t} e_{i}^{\mathrm{T}}(s) \mathrm{H} e_{i}(s) d s \\
& +\frac{1}{2} \sum_{i=1}^{N} \frac{\left(\varsigma_{i}-\widehat{\varsigma}_{i}\right)^{2}}{k_{i}},
\end{aligned}
$$

where $e(t)=\left(\left\|e_{1}(t)\right\|,\left\|e_{2}(t)\right\|, \ldots,\left\|e_{N}(t)\right\|,\left\|e_{1}(t-\tau)\right\|, \| e_{2}(t-\right.$ $\left.\tau)\|, \ldots,\| e_{N}(t-\tau) \|\right)^{\mathrm{T}}, \widehat{\varsigma}_{i}$ and $k_{i}, i=1,2, \ldots, N$, are positive constants, and $H \in \mathbb{R}^{n \times n}$ is the positive definite matrix that needs to be determined. Thus, the time derivative of $V(e, t, \tau)$ along (6) is

$$
\begin{aligned}
\dot{V}(e, t, \tau)= & \frac{1}{2} \sum_{i=1}^{N}\left(\dot{e}_{i}^{\mathrm{T}}(t) e_{i}(t)+e_{i}^{\mathrm{T}}(t) \dot{e}_{i}(t)\right) \\
& +\sum_{i=1}^{N}\left[e_{i}^{\mathrm{T}}(t) \mathrm{H} e_{i}(t)-e_{i}^{\mathrm{T}}(t-\tau) \mathrm{H} e_{i}(t-\tau)\right] \\
& -\sum_{i=1}^{N} \frac{\left(\varsigma_{i}-\widehat{\varsigma}_{i}\right) \dot{\zeta}_{i}}{k_{i}}=\frac{1}{2} \sum_{i=1}^{N}\left[\left(A(t) e_{i}(t)\right.\right. \\
& +B(t) e_{i}(t-\tau)+h_{1}\left(x_{i}, \xi\right)+h_{2}\left(x_{i}(t-\tau), \xi\right) \\
& +g_{i}\left(x_{1}(t-\tau), x_{2}(t-\tau), \ldots, x_{N}(t-\tau)\right) \\
& \left.+u_{i}(t)\right)^{\mathrm{T}} e_{i}(t)+e_{i}^{\mathrm{T}}(t)\left(A(t) e_{i}(t)+B(t) e_{i}(t-\tau)\right. \\
& +h_{1}\left(x_{i}, \xi\right)+h_{2}\left(x_{i}(t-\tau), \xi\right) \\
& +g_{i}\left(x_{1}(t-\tau), x_{2}(t-\tau), \ldots, x_{N}(t-\tau), \xi\right) \\
& +\sum_{i=1}^{N} \frac{\left(\varsigma_{i}-\widehat{\varsigma}_{i}\right) \dot{\zeta}_{i}}{k_{i}} . \\
& \left.\left.+u_{i}(t)\right)\right]+\sum_{i=1}^{N}\left[e_{i}^{\mathrm{T}}(t) \mathrm{H} e_{i}(t)-e_{i}^{\mathrm{T}}(t-\tau) \mathrm{H} e_{i}(t\right. \\
& +2 \mathrm{H}] e_{i}(t)+\sum_{i=1}^{N}\left[e_{i}^{\mathrm{T}}(t-\tau) B_{i}^{\mathrm{T}}(t) e_{i}(t)+e_{i}^{\mathrm{T}}(t)\right. \\
& +\tau)]-\sum_{i=1}^{N} \frac{\left(\varsigma_{i}-\widehat{\varsigma}_{i}\right) \dot{\zeta}_{i}}{k_{i}}=\frac{1}{2} \sum_{i=1}^{N} e_{i}^{\mathrm{T}}(t)\left[e_{i}^{\mathrm{T}}(t-\tau) \mathrm{H} e_{i}(t-\tau)+A(t)\right.
\end{aligned}
$$


The substitution of the adaptive controller (3) into (13) yields the following:

$$
\begin{aligned}
\dot{V} & (e, t, \tau)=\sum_{i=1}^{N} e_{i}^{\mathrm{T}}(t)\left[\frac{A^{\mathrm{T}}(t)+A(t)}{2}+\mathrm{H}\right] e_{i}(t) \\
& +\sum_{i=1}^{N}\left[e_{i}^{\mathrm{T}}(t-\tau) B^{\mathrm{T}}(t) e_{i}(t)+e_{i}^{\mathrm{T}}(t) B(t) e_{i}(t-\tau)\right] \\
& +\sum_{i=1}^{N} e_{i}^{\mathrm{T}}(t) \\
& \cdot g_{i}\left(x_{1}(t-\tau), x_{2}(t-\tau), \ldots, x_{N}(t-\tau), \xi\right) \\
& +\sum_{i=1}^{N} e_{i}^{\mathrm{T}}(t)\left[h_{1}\left(x_{i}, \xi\right)+h_{2}\left(x_{i}(t-\tau), \xi\right)\right] \\
& +\sum_{i=1}^{N} \varsigma_{i} e_{i}^{\mathrm{T}}(t) e_{i}(t)-\sum_{i=1}^{N} e_{i}^{\mathrm{T}}(t-\tau) Q e_{i}(t-\tau) \\
& -\sum_{i=1}^{N}\left(\varsigma_{i}-\widehat{\varsigma}_{i}\right) e_{i}^{\mathrm{T}}(t) e_{i}(t) .
\end{aligned}
$$

According to Assumptions 4 and 7 and Lemma 5, selecting $\mathrm{H}=\varepsilon I_{n \times n}$, where the constant $\varepsilon>0$, the following can be obtained:

$$
\begin{aligned}
\dot{V}(e, t, \tau) \leq \sum_{i=1}^{N} e_{i}^{\mathrm{T}}(t)\left[\frac{A^{\mathrm{T}}(t)+A(t)}{2}\right. & \left.+\left(\varepsilon+\gamma_{i}-\widehat{\varsigma}_{i}\right) I_{n \times n}\right] e_{i}(t)+\frac{1}{2} \\
& +\sum_{i=1}^{N}\left[e_{i}^{\mathrm{T}}(t-\tau)\left(B^{\mathrm{T}} B+I\right) e_{i}(t-\tau)\right. \\
& \left.+e_{i}^{\mathrm{T}}(t)\left(B^{\mathrm{T}} B+I\right) e_{i}(t)\right] \\
& +\sum_{i=1}^{N} \sum_{j=1}^{N}\left(\mu_{i j}+\gamma_{i}\right)\left\|e_{i}(t)\right\|_{2}\left\|e_{j}(t-\tau)\right\|_{2}+\sum_{i=1}^{N} \gamma_{i} \\
& \cdot\left\|e_{i}(t)\right\|_{2}^{2}-\sum_{i=1}^{N} e_{i}^{\mathrm{T}}(t-\tau) \mathrm{H} e_{i}(t-\tau) \leq \sum_{i=1}^{N}(\alpha+\varepsilon \\
& +\beta^{2}+\gamma_{i}+1-\widehat{\varsigma}_{1}, \ldots, \alpha+\varepsilon+\beta^{2}+\gamma_{i}+1-\widehat{\varsigma}_{N} \\
& \left.+\beta^{2}+\gamma_{i}+1-\widehat{\varsigma}_{i}\right)\left\|e_{i}(t)\right\|_{2}^{2} \\
& +\sum_{i=1}^{N} \sum_{j=1}^{N}\left(\mu_{i j}+\gamma_{i}\right)\left\|e_{i}(t)\right\|_{2}\left\|e_{j}(t-\tau)\right\|_{2} \\
& \frac{\beta^{2}-\varepsilon+1}{2} \sum_{i=1}^{N}\left\|e_{i}(t-\tau)\right\|_{2}^{2}=e^{\mathrm{T}}(t)(\operatorname{diag}\{\alpha+\varepsilon \\
&
\end{aligned}
$$

where $\mathrm{P}=\left(\begin{array}{cc}0 & \Gamma / 2 \\ \Gamma / 2 & 0\end{array}\right), \Gamma=\left(\mu_{i j}+\gamma_{i}\right)_{N \times N}$.
Therefore, we can choose the appropriate constants $\widehat{\varsigma}_{i}, i=$ $1,2, \ldots, N$, and $\varepsilon>0$, such that the diagonal matrix $\operatorname{diag}\{\alpha+$ $\varepsilon+\beta^{2}+\gamma_{i}+1-\widehat{\varsigma}_{1}, \ldots, \alpha+\varepsilon+\beta^{2}+\gamma_{i}+1-\widehat{\varsigma}_{N},-\left(\beta^{2}-\right.$ $\left.\varepsilon+1) / 2, \ldots,-\left(\beta^{2}-\varepsilon+1\right) / 2\right\}+P$ is a negative matrix. This equation shows that $\varsigma_{i}, i=1,2, \ldots, N$, is uniformly bounded. By using the Lyapunov method, the error system (18) is also asymptotically stable under the action of the adaptive controller (11) (e.g., $\left.\lim _{t \rightarrow \infty}\left\|e_{i}(t)\right\|=0, i=1,2, \ldots, N\right)$. It is concluded that $e=0$ is an asymptotically stable equilibrium point of the error system (19).

\section{Numerical Simulation}

In this section, two typical examples of the complex network are used to show the correctness and effectiveness of the adaptive controller (11). One example is a ring network with fifty nodes, in which the dynamic performance for each node is complex. The other example is a dynamical network with switching topologies and twelve second-order nodes, in which the dynamic performance for each node is more complex. And the network state equation satisfies (3).

4.1. Simulation I. Chaos often comes from the bifurcation control to the equation of the form

$$
\begin{aligned}
\frac{d x}{d t} & =\text { production }- \text { destruction } \\
& =p g(x(t-\tau))-q x
\end{aligned}
$$

where $g(\cdot)$ is a nonlinear function and $p>0, q>0$. Generally speaking, maximum production occurs at some intermediate value of $x(t-\tau)$, and thus the control displays both positive and negative feedback characteristics. The M-G system [34, 35]

$$
\dot{x}(t)=-p x(t)+q \frac{x(t-\tau)}{1+x(t-\tau)^{r}}
$$

comes from the equation in case of controlling the density of circulating blood cells. We choose this model for our studies since it has been thoroughly investigated in the literature [34] and is easily implementable electronically [35]. The number of positive Lyapunov exponents as well as the dimension of the strange attractor of this system can easily be controlled by varying the delay time $\tau$. For fixed values of the parameters, both of these quantities increase linearly with the increase of $\tau$ [34]. In order to analyze the system dynamics and the proposed controller design, it is adopted in (25) that a polynomial approximation of the M-G system shows the same qualitative behavior. The system equation thus becomes

$$
\dot{x}(t)=-p x(t)+q\left[x(t-\tau)-x^{3}(t-\tau)\right] .
$$

When $p=0.33, q=1.33$, and $\tau=4$, system (27) becomes chaotic. This system has three unstable fixed points:

$$
\begin{aligned}
\bar{x}_{1} & =0, \\
\bar{x}_{2,3} & = \pm\left(1-\frac{p}{q}\right)^{1 / 2} .
\end{aligned}
$$


We choose a ring network with fifty nodes as our complex network, because the network is one regular network of all typical networks and is an ordinary mathematical model [36], which can represent many physical models. Assume that it satisfies the following conditions:

(1) The complex network coupling function form is

$$
\begin{aligned}
g_{i} & \left(x_{1}(t-\tau), x_{2}(t-\tau), \ldots, x_{N}(t-\tau)\right) \\
& =\sum_{j=1}^{N} \mu_{i j} x_{j}(t-\tau) .
\end{aligned}
$$

(2) It satisfies the previous conditions in Assumptions 3 and 4 .

(3) The dynamic equations of each node are M-G systems, where $N=50$, and the coupling strengths are all 0.1 .

The network structure is shown in Figure 1.

Then, the state equation of this complex network can be written as

$$
\begin{aligned}
& \dot{x}_{1}(t)=-0.33 x_{1}(t)+1.33\left[x_{1}(t-\tau)-x_{1}^{3}(t-\tau)\right] \\
& +0.1 x_{2}(t)+0.1 x_{50}(t)+u_{1}(t), \\
& \dot{x}_{2}(t)=-0.33 x_{2}(t)+1.33\left[x_{2}(t-\tau)-x_{2}^{3}(t-\tau)\right] \\
& +0.1 x_{1}(t)+0.1 x_{3}(t)+u_{2}(t), \\
& \dot{x}_{3}(t)=-0.33 x_{3}(t)+1.33\left[x_{3}(t-\tau)-x_{3}^{3}(t-\tau)\right] \\
& +0.1 x_{2}(t)+0.1 x_{4}(t)+u_{3}(t), \\
& \quad \vdots \\
& \dot{x}_{50}(t)=-0.33 x_{50}(t) \\
& +1.33\left[x_{50}(t-\tau)-x_{50}^{3}(t-\tau)\right]+0.1 x_{1}(t) \\
& +0.1 x_{49}(t)+u_{4}(t) .
\end{aligned}
$$

From the knowledge of Section 2, it is well known that such networks are very hard to synchronize. Figure 2 shows the state responses of this ring complex network with $\tau=$ 0.5 and without control. It is obvious that the network is desynchronized.

The synchronization target node is selected as $x=$ $\sqrt{1-p / q} \doteq 0.867$. The initial states of 50 nodes are selected randomly from 0 to 25 . Let $k_{i}=25, \varsigma_{i}(0)=$ 10 , and then according to Theorem 8 , it can be seen that this complex network is locally asymptotically synchronous under the action of adaptive controller (11). The adaptive synchronization analysis of complex networks is carried out by using MATLAB to prove that the adaptive controller can synchronize the complex network on the trajectory of the specified chaotic node. If the simulation result is correct, this will prove that the controller is effective. Figures 3 and 4 show the synchronous error $e_{i}, i=1,2, \ldots, 50$, for $\tau=0.1$ and $\tau=0.5$ with the same controller, respectively.

Obviously, it should be pointed out that the controlled network is asymptotically stable at zero under the effective

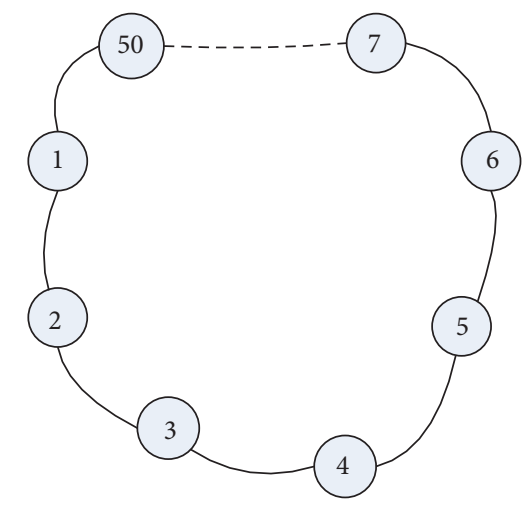

FIGURE 1: The ring network structure with 50 nodes.

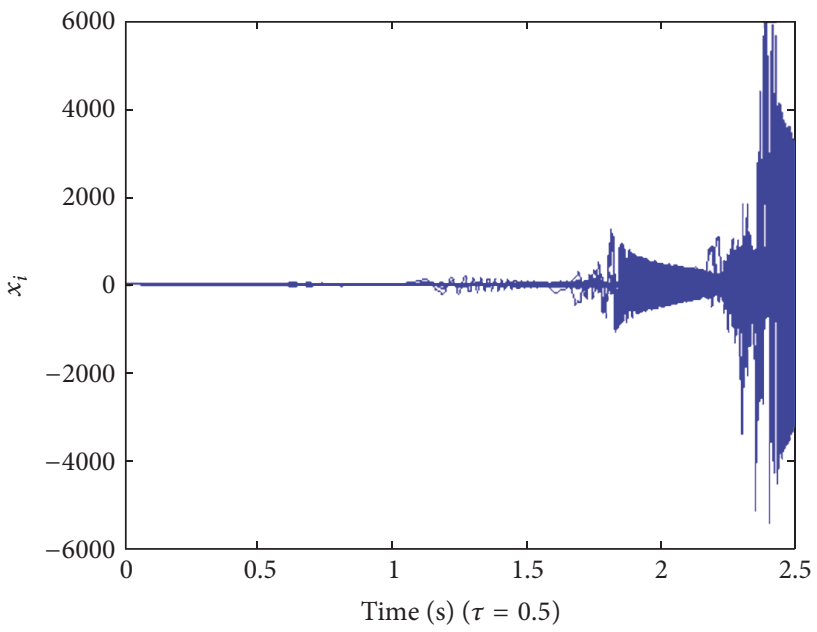

FIGURE 2: State response $x_{i}$ of 50 nodes' dynamical network without control.

adaptive controller (11). The simulation results indicate that the coupling delays create some obstacle to the synchronization of the complex networks. The response performance is deteriorated as the time delays become large. It is clear from the figures that the errors are very small.

4.2. Simulation II. The proposed theorems have been used for synchronizing the second-order nodes in the dynamical complex network. Consider twelve second-order nodes in the dynamical network with switching topologies. The state equation of each second-order node is described by

$$
M_{i} \ddot{x}_{i}=u_{i}
$$

that is,

$$
\begin{aligned}
\dot{x}_{i} & =v_{i}, \\
M_{i} \dot{v}_{i} & =u_{i},
\end{aligned}
$$

where $x_{i} \in \mathbb{R}^{m}$ are the position vectors; $v_{i} \in \mathbb{R}^{m}$ stand for the velocity vectors; $M_{i} \in \mathbb{R}^{m \times m}$ are symmetric positive definite matrices; and $u_{i} \in \mathbb{R}^{m}$ are the control inputs. Let $M=$ $\operatorname{diag}\left\{M_{1}, M_{2}, \ldots, M_{n}\right\} \in \mathbb{R}^{n m \times n m}, x=\left[x_{1}^{\mathrm{T}}, x_{2}^{\mathrm{T}}, \ldots, x_{n}^{\mathrm{T}}\right]^{\mathrm{T}} \in$ 


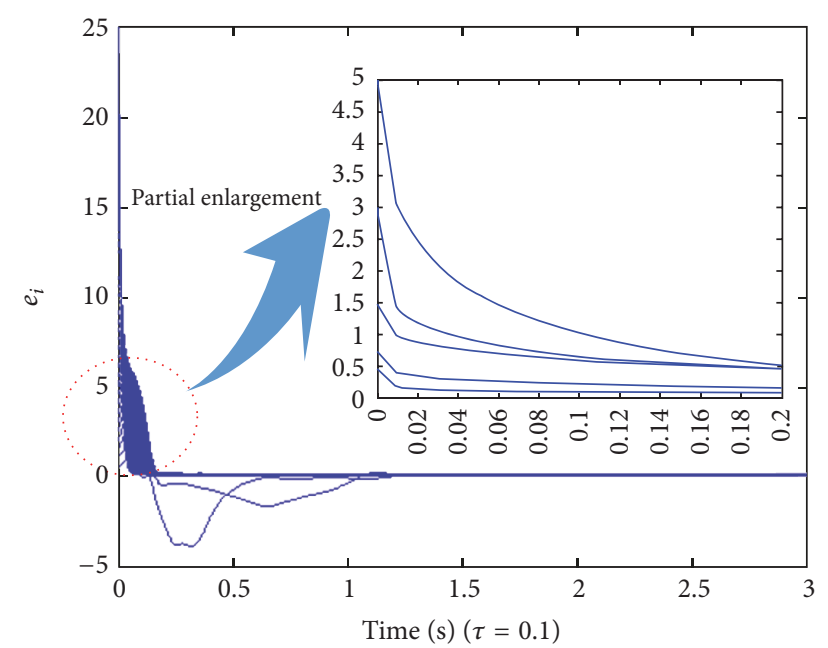

FIGURE 3: Synchronization error $e_{i}$ of 50 nodes' network with $\tau=0.1$.

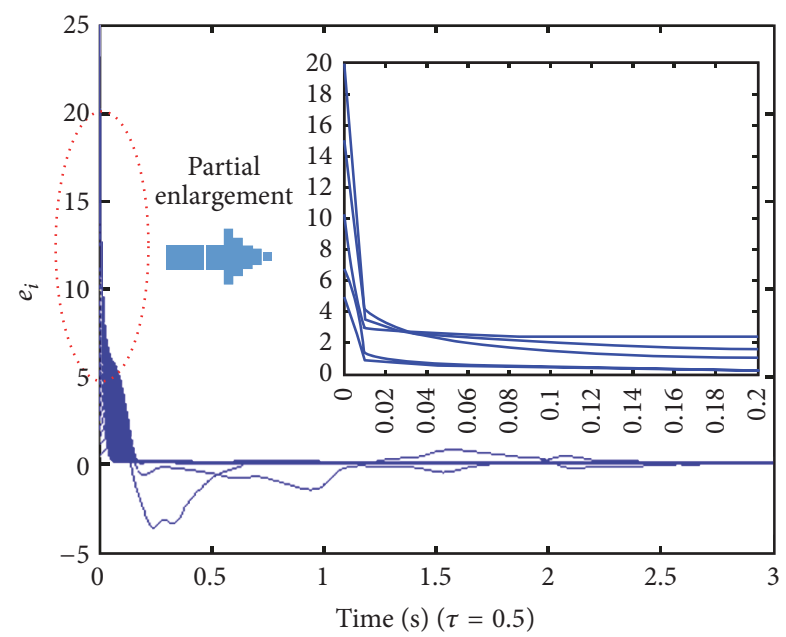

FIGURE 4: Synchronization error $e_{i}$ of 50 nodes' network with $\tau=$ 0.5 .

$\mathbb{R}^{n m}, v=\left[v_{1}^{\mathrm{T}}, v_{2}^{\mathrm{T}}, \ldots, v_{n}^{\mathrm{T}}\right]^{\mathrm{T}} \in \mathbb{R}^{n m}$, and $u=\left[u_{1}^{\mathrm{T}}, u_{2}^{\mathrm{T}}, \ldots, u_{n}^{\mathrm{T}}\right]^{\mathrm{T}} \in$ $\mathbb{R}^{n m}$. According to (32), for the arbitrary $n$th node, there is

$$
\begin{aligned}
\dot{x} & =v, \\
M \dot{v} & =u .
\end{aligned}
$$

Suppose that there exists a communication time delay $\tau$ between nodes $i$ and $j$. The interaction topology of a dynamical network of nodes with second-order nodes is represented using a directed graph $G=(V, E, A)$ with the set of nodes $V=\left\{v_{1}, v_{2}, \ldots, v_{n}\right\}$, the set of directed edges $E \subseteq V \times V$, and the adjacency matrix $A=\left[a_{i j}\right] \in \mathbb{R}^{n \times n}$. $\mathrm{H} \in\{1,2, \ldots, n\}$ is the set of node subscripts, and $e_{i j}=(i, j)$ denotes the directed edge from node $i$ to node $j$. The elements in the adjacency matrix $A=\left[a_{i j}\right] \in \mathbb{R}^{n \times n}$ satisfy $a_{i i}=0$, $a_{i j}>0$ (if and only if $e_{i j} \in E$ ). The set of neighbors of a node $i$ is $\aleph_{i}=\{j \in \mathrm{H}:(i, j) \in E\}$. Let $d_{i}=\sum_{j \in \aleph_{i}} a_{i j}, i \in \mathrm{H}$; the degree matrix of a directed graph $G$ can be expressed as $D=\operatorname{diag}\left\{d_{1}, d_{2}, \ldots, d_{n}\right\}$. The Laplacian matrix $L$ is defined by $L \triangleq D-A$. It can be shown that, using the Gershgorin disc theorem [14], all of the eigenvalues of $L$ have a nonnegative real part. Furthermore, if $G$ is undirected, then the Laplacian matrix of $G$ is symmetric and satisfies $L=B B^{\mathrm{T}}$, which means that $L$ is positive semidefinite. The in-degree and out-degree of node $V_{i}$ can be defined as $d_{\text {in }}\left(\nu_{i}\right)=\sum_{j=1}^{n} a_{j i}$ and $d_{\text {out }}\left(\nu_{i}\right)=$ $\sum_{j=1}^{n} a_{i j}$, respectively. If $d_{\text {in }}\left(v_{i}\right)=\sum_{j=1}^{n} a_{j i}=d_{\text {out }}\left(v_{i}\right)=$ $\sum_{j=1}^{n} a_{i j}$, then node $v_{i}$ is an equilibrium point. If there exists a direct graph between nodes in graph $G$, then $G$ is a strongly connected and balanced graph.

Figure 5 gives three strongly connected and balanced graphs with $0-2$ weights. The initial values of $x_{i}$ and $v_{i}$ are selected randomly in the region and $[0,800] \times[0,800]$ and $[0,800] \times[0,800]$, respectively.

The inertias of the twelve second-order nodes are $M_{1}=\operatorname{diag}\{1,1,1,1\}, M_{2}=\operatorname{diag}\{2,2,2,2\}, \ldots, M_{12}=$ $\operatorname{diag}\{12,12,12,12\}$. We set $\tau=0.2$; Figures 6 and 7 show the numerical simulation results of the twelve second-order nodes in the dynamical network with an arbitrary switching signal. Figure 6 gives the inertial nodes' position error curves. Figure 7 shows the inertial nodes' velocity error curves. It is obvious that, for the dynamical network with communication time delay and switching topology, the control strategy proposed in this paper also achieves the exponential stability.

From the above, the synchronization process of the dynamical network can be described as follows: detect the designated node and the other nodes; then, the state variables of nodes are subtracted from the designed node, respectively, by using the subtraction device. This way, we can get the error signals $e_{i}(t)$, and then the error signals are sent to the adaptive controller. Furthermore, the adaptive controller chooses a one-dimensional variable as the control variable. According to $e_{i}(t)$, we can adjust the control outputs and make the variables of the designated node and other nodes synchronized. At the moment, the error $e_{i}(t)$ is close to zero. A block diagram is expressed in Figure 8.

\section{Conclusions}

This paper studied the adaptive synchronization of uncertain dynamical networks with time-delayed nodes and coupling delays, with known or unknown but bounded nonlinear coupling functions. The parameters of the adaptive controllers can be chosen according to the presented theorems which can stabilize the network to the desired fixed points. The adaptive synchronization criteria by using the LyapunovKrasovskii stability theory also provide some new insights into the network synchronization and the possible applications in engineering systems. At last, an example of a dynamical network with fifty M-G system nodes has been simulated, demonstrating the effectiveness of the proposed controllers. Note that the adaptive controllers proposed are both delay-dependent, so there are no compensations to the delay couplings. However, how to solve the delaydependent problem to reach synchronization seems to be new and useful. Furthermore, as another issue, in some real complex systems, interacting subjects are not identical and have different individual properties. Some synchronization 


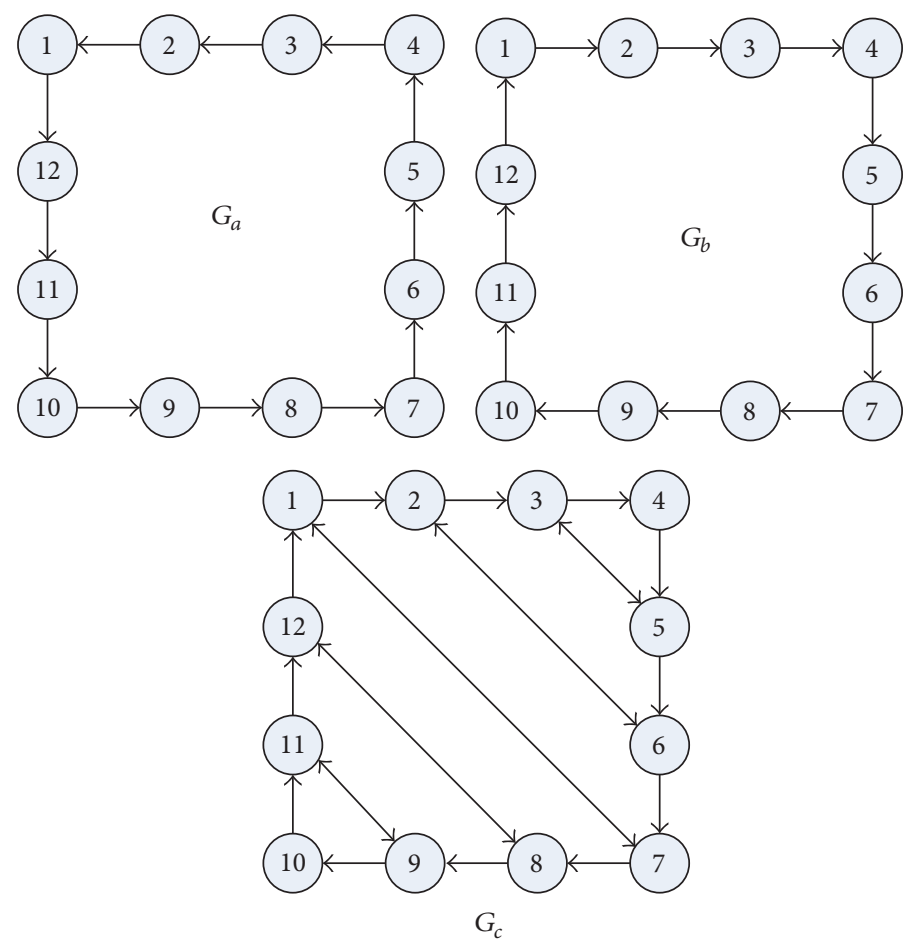

FIGURE 5: Strongly connected and balanced graphs of twelve nodes' network.

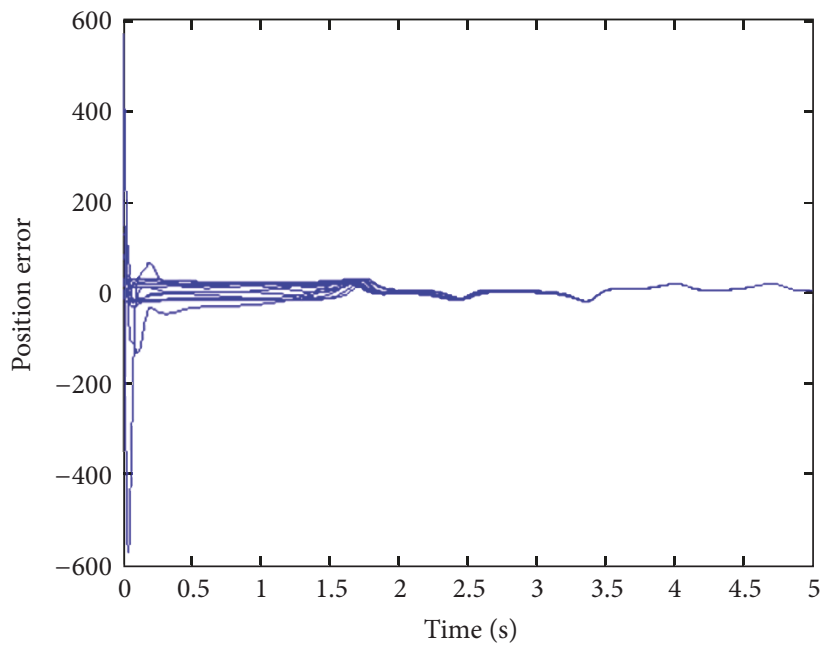

FIGURE 6: The position error curves of twelve second-order nodes' network.

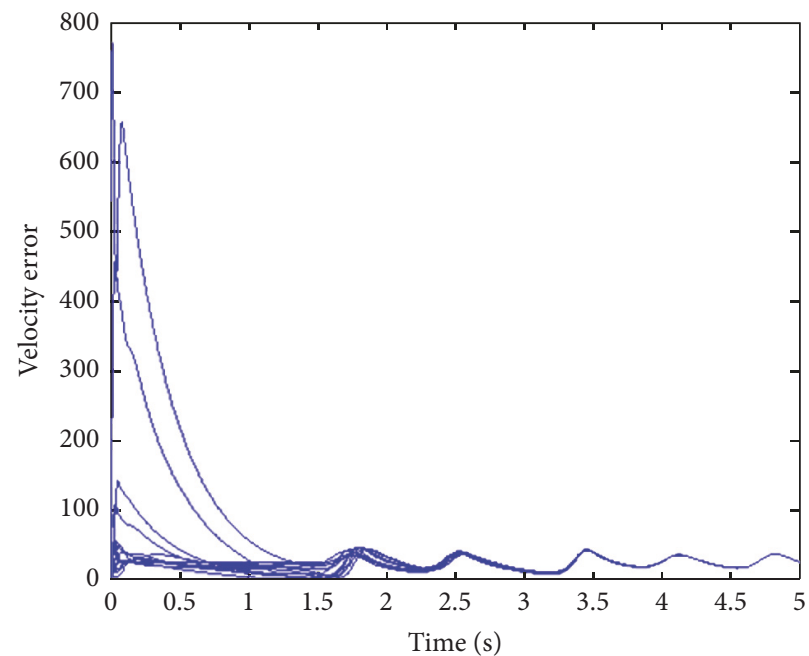

FIGURE 7: The velocity error curves of twelve second-order nodes' network. 


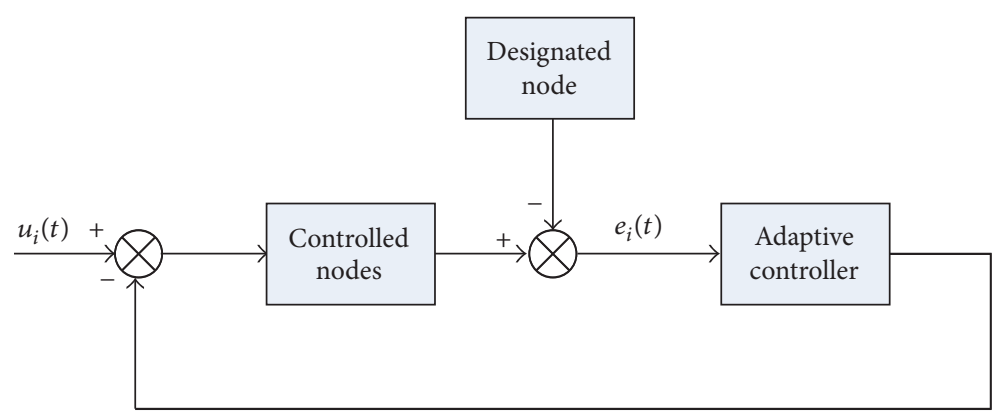

FIgURE 8: The block diagram of adaptive control of the dynamical network.

criteria are expected to be more powerful and deserve further exploration.

\section{Conflicts of Interest}

The authors declare that there are no conflicts of interest regarding the publication of this paper.

\section{Acknowledgments}

The work of Miao Yu was supported by the National Natural Science Foundation of China (51407201, 61402032) and Doctoral Foundation of Beijing University of Civil Engineering and Architecture under Grant 00331614032.

\section{References}

[1] S. H. Strogatz, "Exploring complex networks," Nature, vol. 410, no. 6825 , pp. 268-276, 2001.

[2] M.-J. Mei, K. Nie, D.-S. Xiong, Y.-H. Zhang, and L.-J. Wang, "Research progress of multimodal MRI and complex network analysis based on graph theory in Parkinson's disease," Chinese Journal of Contemporary Neurology and Neurosurgery, vol. 17, no. 1, pp. 14-17, 2017.

[3] X. Sun, H. An, X. Gao, X. Jia, and X. Liu, "Indirect energy flow between industrial sectors in China: a complex network approach," Energy, vol. 94, pp. 195-205, 2016.

[4] F. Serinaldi and C. G. Kilsby, "Irreversibility and complex network behavior of stream flow fluctuations," Physica A: Statistical Mechanics and its Applications, vol. 450, pp. 585-600, 2016.

[5] L. Zhao, G. Diao, P. Yan, Y. Yao, and H. Chen, "A gene recombination method for machine tools design based on complex network," The International Journal of Advanced Manufacturing Technology, vol. 83, no. 5-8, pp. 729-741, 2016.

[6] G. E. Garza, C. C. Posadas, and L. A. Rodriguez, "Chaotic synchronization of irregular complex network with hysteretic circuit-like oscillators in hamiltonian form and its application in private communications," Revista Mexicana de Fisica, vol. 62, no. 1, pp. 51-59, 2016.

[7] L. Ling, C. Li, L. Chen, and G. Zhao, "New technology of synchronization for the uncertain dynamical network with the switching topology," Nonlinear Dynamics, vol. 86, no. 1, pp. 655666, 2016.
[8] W. Shen, Z. Zeng, and S. Wen, "Synchronization of complex dynamical network with piecewise constant argument of generalized type," Neurocomputing, vol. 173, pp. 671-675, 2016.

[9] J. Lehnert, Controlling synchronization patterns in complex networks, Springer International Publishing, Berlin, Germany, 2016.

[10] M. J. Hajikhani, T. Kunz, and H. Schwartz, "A recursive method for clock synchronization in asymmetric packet-based networks," IEEE/ACM Transactions on Networking, vol. 24, no. 4, pp. 2332-2342, 2016.

[11] J. Chen, M. Yu, L.-H. Dou, and M.-G. Gan, "A fast averaging synchronization algorithm for clock oscillators in nonlinear dynamical network with arbitrary time-delays," Acta Automatica Sinica, vol. 36, no. 6, pp. 873-880, 2010.

[12] M. Yu, J. Chen, L.-H. Dou, and M.-G. Gan, "New method of clock synchronization among inverse GPS base-stations," Systems Engineering and Electronics, vol. 31, no. 7, pp. 1710-1714, 2009.

[13] G. Guido, V. Gallelli, D. Rogano, and A. Vitale, "Evaluating the accuracy of vehicle tracking data obtained from Unmanned Aerial Vehicles," vol. 5, pp. 136-151, 2016.

[14] R. Shorten, F. Wirth, and M. Akar, "On nonlinear AIMD congestion control for high-speed networks," in Proceedings of the 45th IEEE Conference on Decision and Control 2006, CDC, pp. 633-638, usa, December 2006.

[15] C. Posadas-Castillo, E. Garza-González, D. A. Diaz-Romero, E. Alcorta-García, and C. Cruz-Hernández, "Synchronization of irregular complex networks with chaotic oscillators: hamiltonian systems approach," Journal of Applied Research and Technology, vol. 12, no. 4, pp. 782-791, 2014.

[16] N. Fujiwara, J. Kurths, and A. Díaz-Guilera, "Synchronization of mobile chaotic oscillator networks," Chaos: An Interdisciplinary Journal of Nonlinear Science, vol. 26, no. 9, Article ID 094824, 094824, 8 pages, 2016.

[17] M. A. Lopes, E. M. Lopes, S. Yoon, J. F. F. Mendes, and A. V. Goltsev, "Synchronization in the random-field Kuramoto model on complex networks," Physical Review E: Statistical, Nonlinear, and Soft Matter Physics, vol. 94, no. 1, Article ID 012308, 2016.

[18] V. Berec, "Explosive synchronization in clustered scale-free networks: revealing the existence of chimera state," The European Physical Journal Special Topics, vol. 225, no. 1, pp. 7-15, 2016.

[19] P. Li, K. Zhang, X. Xu, J. Zhang, and M. Small, "Reexamination of explosive synchronization in scale-free networks: the effect of disassortativity," Physical Review E: Statistical, Nonlinear, and Soft Matter Physics, vol. 87, no. 4, Article ID 042803, 2013. 
[20] J. Zhang, Z. Ma, and J. Cao, "Hub-induced synchronization in scale-free networks with cluster structure," Abstract and Applied Analysis, vol. 2014, no. 2014, Article ID 149453, 7 pages, 2014.

[21] J. Zhou, J.-a. Lu, and J. Lü, "Adaptive synchronization of an uncertain complex dynamical network," Institute of Electrical and Electronics Engineers Transactions on Automatic Control, vol. 51, no. 4, pp. 652-656, 2006.

[22] Q. Li, B. Shen, J. Liang, and H. Shu, "Event-triggered synchronization control for complex networks with uncertain inner coupling," International Journal of General Systems, vol. 44, no. 2, pp. 212-225, 2015.

[23] L. Zhou, C. Wang, and L. Zhou, "Cluster synchronization on multiple sub-networks of complex networks with nonidentical nodes via pinning control," Nonlinear Dynamics, vol. 83, no. 1-2, pp. 1079-1100, 2016.

[24] M. Yu, W. Shang, and Z. Chen, "Exponential synchronization for second-order nodes in complex dynamical network with communication time delays and switching topologies," Journal of Control Science and Engineering, vol. 2017, Article ID 7836316, pp. 1-10, 2017.

[25] L.-X. Li, H.-P. Peng, B.-Z. Guan, and J.-M. Xu, "A new sliding mode control for a class of uncertain time-delay chaotic systems," Chinese Physics, vol. 10, no. 8, pp. 708-710, 2001.

[26] L.-X. Li, H.-P. Peng, H.-B. Lu, and X.-P. Guan, "On feedback control of delayed chaotic system," Chinese Physics, vol. 10, no. 9, pp. 796-804, 2001.

[27] M. Dhamala, V. K. Jirsa, and M. Ding, "Enhancement of neural synchrony by time delay," Physical Review Letters, vol. 92, no. 7, article no 074104, 2004.

[28] F. M. Atay, J. Jost, and A. Wende, "Delays, connection topology, and synchronization of coupled chaotic maps," Physical Review Letters, vol. 92, no. 14, article no 144101, 2004.

[29] Y. Gao, L. X. Li, H. P. Peng, Y. . Yang, and X. H. Zhang, "Stability analysis of complex networks with multi-links," Acta Physica Sinica, vol. 57, no. 3, pp. 1444-1452, 2008.

[30] X. Fang and W. Chen, "Synchronization of complex dynamical networks with time-varying inner coupling," Nonlinear Dynamics, vol. 85, no. 1, pp. 13-21, 2016.

[31] C. Li and G. Chen, "Synchronization in general complex dynamical networks with coupling delays," Physica A: Statistical Mechanics and its Applications, vol. 343, no. 1-4, pp. 263-278, 2004.

[32] V. A. Yakubovich, "Linear matrix inequalities in system and control theory," SIAM Review, vol. 37, no. 3, pp. 479-481, 2012.

[33] Z. Li and G. Chen, "Robust adaptive synchronization of uncertain dynamical networks," Physics Letters A, vol. 324, no. 2-3, pp. 166-178, 2004.

[34] J. D. Farmer, "Chaotic attractors of an infinite-dimensional dynamical system," Physica D: Nonlinear Phenomena, vol. 4, no. 3, pp. 366-393, 1982.

[35] A. Namajunas, K. Pyragas, and A. Tamaševičius, "An electronic analog of the Mackey-Glass system," Physics Letters A, vol. 201, no. 1, pp. 42-46, 1995.

[36] H. G. Tanner, A. Jadbabaie, and G. J. Pappas, "Flocking in fixed and switching networks," Institute of Electrical and Electronics Engineers Transactions on Automatic Control, vol. 52, no. 5, pp. 863-868, 2007. 


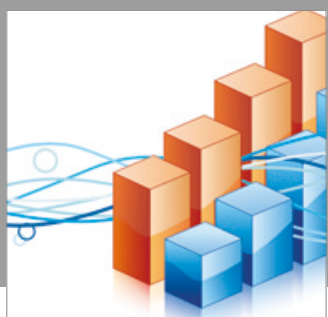

Advances in

Operations Research

vatersals

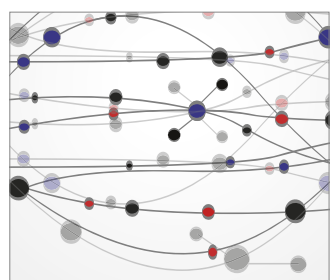

\section{The Scientific} World Journal
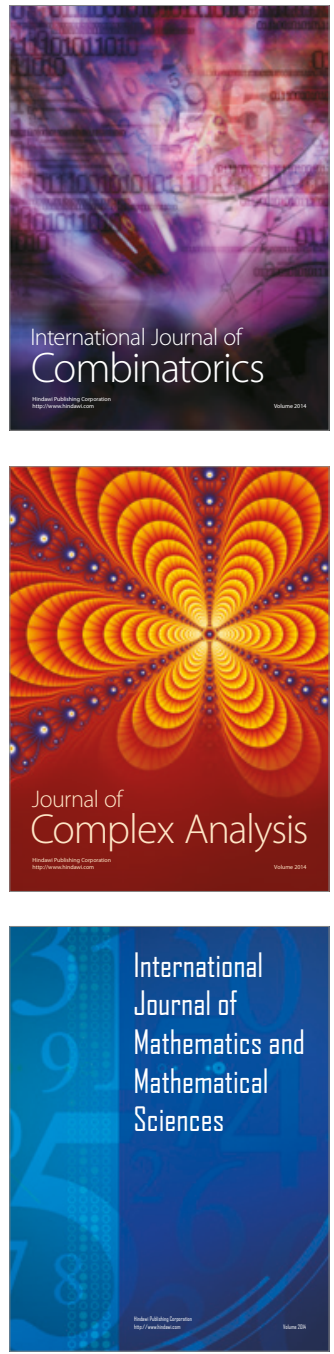
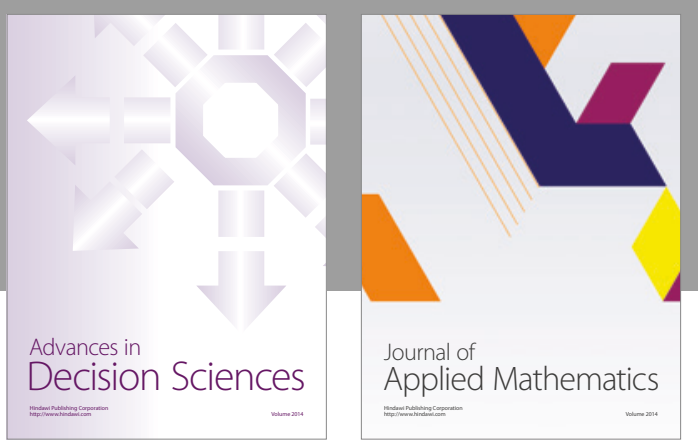

Algebra

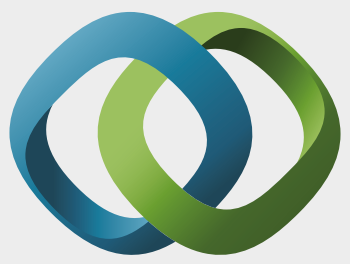

\section{Hindawi}

Submit your manuscripts at

https://www.hindawi.com
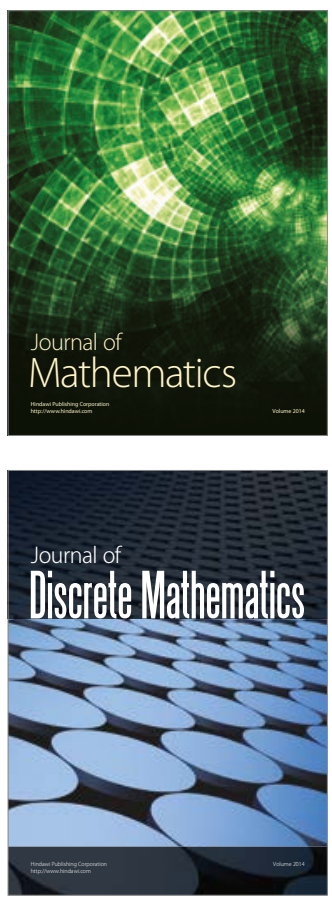

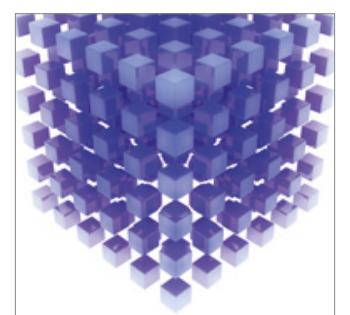

Mathematical Problems in Engineering
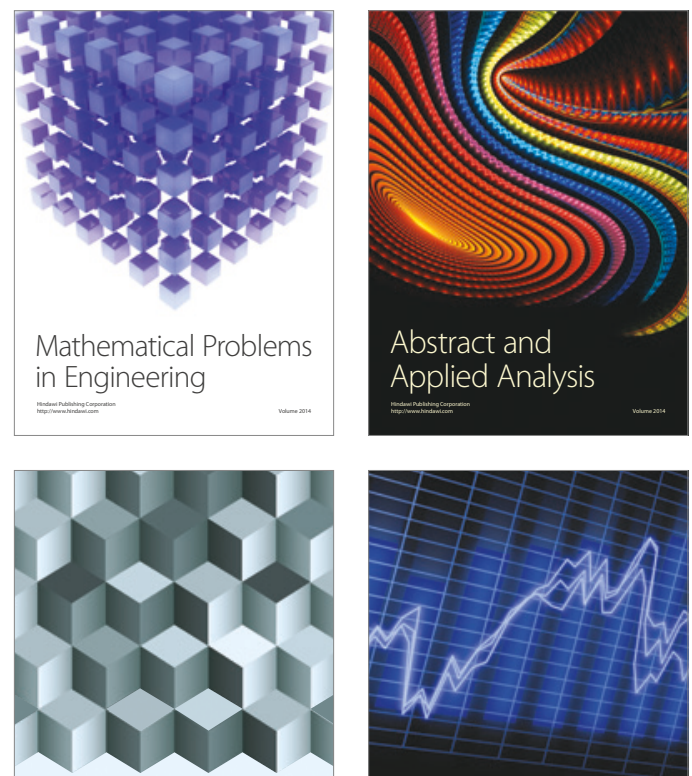

Journal of

Function Spaces

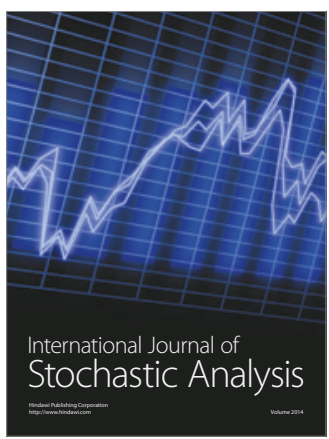

Probability and Statistics
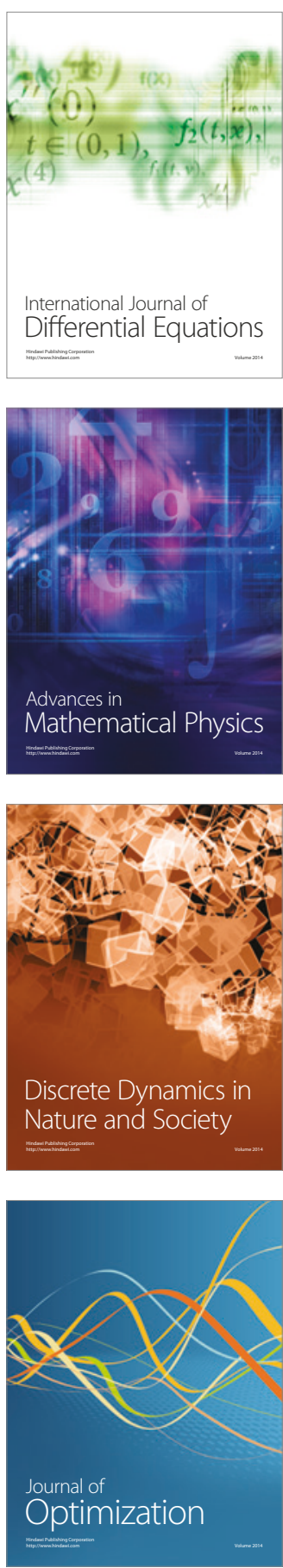